\title{
Étude de l'évolution de la composition des lactosérums au cours de leur conservation
}

\author{
par \\ Annie IMBERT-PONDAVEN*
}

\section{INTRODUCTION}

Le besoin mondial en protéines s'accroît plus vite que le besoin alimentaire global. La production de protéines est marquée par une rareté véritable, même catastrophique dans beaucoup de pays du "Tiers Monde ». Dans les pays riches, elle se caractérise par une consommation croissante répondant plus à une surchage qu'à une nécessité [24].

Particulièrement intéressantes sont les protéines du soja et du lait. Toutes les deux possèdent presque la même valeur nutritive. Le soja ne peut être cultivé que dans certaines zones climatiques, ce qui limite son extension. La production du lait peut se développer dans les régions de pâturages qui constituent environ 60 p. 100 de la surface cultivable de la terre.

En France, la production de lait s'est accrue de 74 p. 100 au cours de ces 10 dernières années [18]. Ceci a amené les industriels laitiers à valoriser les sous-produits comme le lactosérum de fromagerie à la fois pour des raisons de rentabilité et de lutte contre la pollution.

Le lactosérum, sous-produit de la fabrication du fromage ou de la caséine, peut être défini comme étant un lait privé de caséine. L'intérêt porté à ce produit dépend essentiellement de la fraction protéique, du lactose et des vitamines hydrosolubles qu'il renferme. Les autres constituants (sels minéraux et acide lactique) sont considérés très souvent comme des facteurs limitant l'utilisation. Cepen-

* Ministère de l'Agriculture, Direction de la Qualité, Services Vétérinaires. Laboratoire Central d'Hygiène Alimentaire, 43, rue de Dantzig - 75015 Paris.

(Directeur : J. Pantaléon). 
dant, les traitements physiques actuels (osmose inverse, ultrafiltration et électrodialyse) permettent de moduler la composition du produit.

A chaque type de fromage correspond un lactosérum particulier. Il est donc impossible, du fait de la grande variété des fromages d'admettre une composition standard.

Pour réaliser la présente étude, un choix a été nécessaire. Nous avons suivi les modifications de certains composants, principalement la fraction azotée non protéique, dans deux types distincts de lactosérum provenant l'un de la préparation de fromage à pâte fraîche à partir d'un caillé lactique, l'autre de la préparation de fromage à pâte pressée à partir d'un caillé présure.

L'influence de la température a été évaluée après $5 \mathrm{j}$ de conservation du produit liquide. La température de stockage est généralement inférieure à $+10^{\circ} \mathrm{C}$ en industrie, mais elle peut atteindre des valeurs supérieures si le sérum n'est pas refroidi après la fabrication. Nous avons donc choisi deux températures :

$+4^{\circ} \mathrm{C}$ : température habituellement conseillée pour l'entreposage ;

$+23^{\circ} \mathrm{C}$ : température ambiante ;

et pour certains critères, la température de $+10^{\circ} \mathrm{C}$ fut retenue.

Les lactosérums étudiés [39] proviennent de fabrications réalisées au laboratoire à partir de lait cru*. Ils ont été analysés dès leur obtention et après $5 \mathrm{j}$ de conservation à $+4^{\circ} \mathrm{C},+10^{\circ} \mathrm{C},+23^{\circ} \mathrm{C}$. Le lactosérum acide est obtenu après acidification lente du lait sous l'influence de ferments lactiques et séparation du caillé. Le lactosérum doux est le liquide d'exsudation obtenu lors de la fabrication des fromages à pâte cusite ou pressée.

\section{ETUDE DE QUELQUES COMPOSANTS A L'EXCLUSION DES PROTIDES}

Par comparaison avec le lait, le lactosérum est un produit pauvre en matière sèche ; celle-ci est représentée principalement par le lactose. Sa teneur en matières protéiques est faible mais il est riche en matières minérales. Le tableau 1 résume quelques données extraites de la littérature $[5,10,15]$.

\footnotetext{
* Le lait utilisé pour les fabrications provenait du troupeau de vaches de race française Frisonne Pie Noire du domaine expérimental de l'I.N.R.A. à Bressonvillers, acheminé en camion citerne à la Laiterie Expérimentale de l'I.N.R.A. à Jouy-en-Josas.
} 
TABLEAU 1. - Composition comparée de quelques lactosérums (p. 100)

\begin{tabular}{|c|c|c|c|c|c|c|}
\hline & $\underset{(1)}{\text { Emmental }}$ & $\begin{array}{l}\text { Gruyère } \\
\text { (1) }\end{array}$ & $\begin{array}{l}\text { Présure } \\
\text { (2) }\end{array}$ & $\underset{(3)}{\text { Emmental }}$ & $\begin{array}{l}\text { Acide } \\
(2)\end{array}$ & Pâte fraîche \\
\hline Matière sèche & $6,41-6,60$ & $6,85-6,96$ & $6,0-7,0$ & $7,0 \quad-7,3$ & $5,0-6,0$ & $6,4 \quad-6,5$ \\
\hline Protéines totales & $0,65-0,73$ & $0,67-0,73$ & $0,8-1,0$ & $0,86-0,93$ & $0,8-1,0$ & $0,6 \quad-0,79$ \\
\hline Matière grasse & $0,35-0,56$ & $0,41-0,59$ & 0,1 & $0,5 \quad-0,6$ & traces & $0,05-0,09$ \\
\hline Lactose & $\begin{array}{ll}4,59 & -4,79\end{array}$ & $4,82-5,05$ & $4,5-4,8$ & $5,07-5,31$ & $3,8-4,2$ & $4,34 \quad-4,47$ \\
\hline Cendres & $0,48 \quad-0,59$ & $0,51-0,56$ & $0,5-0,7$ & $0,52-0,53$ & $0,7-0,8$ & $0,73-0,76$ \\
\hline Calcium & $0,033-0,0370$ & $0,034-0,038$ & - & $0,043-0,044$ & - & $0,128-0,138$ \\
\hline Acide lactique & $0,04-0,10$ & $0,03-0,12$ & traces & $0,03-0,08$ & 0,8 & $0,78-0,80$ \\
\hline
\end{tabular}

(1) BLANC, 1969 [5].

(2) Doose, 1963 (cité par Collet) [10].

(3) FÉVRIER, 1972 [15]. 


\section{A. Teneur en matière sèche}

La teneur en matière sèche des lactosérums est faible puisqu'elle ne représente que 6 à 7 p. 100. Ceci constitue l'une des caractéristiques essentielles du point de vue de sa manipulation, de son traitement et de sa valeur nutritive. Contrairement aux résultats généralement trouvés dans la littérature, la teneur en matière sèche du sérum acide, analysé dès l'obtention, est supérieure à celle du lactosérum doux. Tarodo de La Fuente [50] explique ce phénomène par une différence de déshydratation des molécules ou des micelles de caséine : elle se poursuit par une synérèse faible dans le caillé de type acide et beaucoup plus importante dans le caillé de type présure. L'examen du tableau 2 indique que la teneur en matière sèche diminue d'autant plus que la température de conservation des deux types de sérum est élevée.

\section{B. Teneur en lactose}

Les résultats de la détermination de la teneur en lactose, par polarimétrie [46] indiquent une diminution importante des glucides dans le lactosérum doux conservé à température ambiante (tab. 2). Dans le lactosérum acide, la teneur est nettement plus faible et ceci dès son obtention. Au cours de la conservation des sérums liquides, I'hydrolyse du lactose en glucose et galactose s'ajoute aux processus fermentaires. A l'analyse, la présence de galactose dont l'angle de déviation du plan de polarisation de la lumière est supérieur à celui du lactose, fausse par excès les résultats trouvés.

\section{Acidité titrable et $\mathrm{pH}$}

Les deux types de lactosérum présentent des différences notables. Les valeurs de $\mathrm{pH}$ et d'acidité titrable ne sont pas étroitement liées, le $\mathrm{pH}$ ne s'abaisse pas proportionnellement à l'élévation de l'acidité titrable. Mais dans le cas du sérum frais de fromage à pâte cuite, l'acidité est basse du fait de la coagulation du phosphocaséinate, le $\mathrm{pH}$ est peu différent de celui du lait d'origine (tab. 2). En revanche, dans le sérum de caillé acide, l'acidité titrable est élevée : elle représente la somme de l'acidité développée et d'une partie de l'acidité naturelle due aux phosphates, le pH est bas.

$\mathrm{A}+4^{\circ} \mathrm{C}$, le développement des germes psychrotrophes tend à alcaliniser le milieu. Après un séjour aux températures plus élevées $\left(+10^{\circ} \mathrm{C}\right.$ et $\left.+23^{\circ} \mathrm{C}\right)$, le lactosérum doux s'acidifie, alors que le lactosérum acide ne subit pas d'augmentation sensible de l'acidité.

\section{Teneur en matières minérales}

La teneur moyenne en minéraux des lactosérums acide et doux analysés est respectivement égale à 11,5 et $7,5 \mathrm{~g}$ pour $100 \mathrm{~g}$ de matière sèche. Cette différence s'explique par le mode de coagulation de la caséine $[39,50]$. 
TABLEAU 2. - Matière sèche, lactose, acidité titrable et $\mathrm{pH}$ des lactosérums acide et doux

\begin{tabular}{|c|c|c|c|c|c|c|c|c|}
\hline \multirow{2}{*}{ Conservation } & \multicolumn{2}{|c|}{$\begin{array}{c}\text { Teneur en matière } \\
\text { sèche } \\
(\mathrm{g} \mathrm{p} .100 \mathrm{ml})\end{array}$} & \multicolumn{2}{|c|}{$\begin{array}{l}\text { Teneur en lactose } \\
(\mathrm{g} \text { p. } 100 \mathrm{~g} \mathrm{MS})\end{array}$} & \multicolumn{2}{|c|}{$\begin{array}{c}\text { Acidité titrable } \\
\text { (g acide lactique) } \\
(\text { p } 100 \mathrm{ml})\end{array}$} & \multicolumn{2}{|c|}{$\begin{array}{c}\text { Acidité ionique } \\
\text { ou } \mathrm{pH}\end{array}$} \\
\hline & acide & doux & acide & doux & acide & doux & acide & doux \\
\hline Dès l'obtention & 6,85 & 6,61 & 58,2 & 76,9 & 0,546 & 0,096 & 4,61 & 6,55 \\
\hline Après $5 \mathrm{j}$ à $+4^{\circ} \mathrm{C}$ & 6,83 & 6,57 & 61,7 & 74,8 & 0,550 & 0,094 & 4,63 & 6,64 \\
\hline Après $5 \mathrm{j}$ à $+10^{\circ} \mathrm{C}$ & 6,78 & 6,55 & 61,7 & 71,7 & 0,547 & 0,195 & 4,61 & 5,43 \\
\hline Après $5 \mathrm{j}$ à $+23^{\circ} \mathrm{C}$ & 6,40 & 6,25 & 63,3 & 64,8 & 0,594 & 0,451 & 4,59 & 4,08 \\
\hline
\end{tabular}




\section{MATIERES AZOTEES DES LACTOSERUMS ACIDE ET DOUX}

La teneur moyenne en matière azotée des lactosérums varie de 11 à 13 p. 100 de matière sèche. Les composés protidiques sont représentés par 70 à 75 p. 100 des protéines solubles (albumines - globulines - protéoses peptones - protéines mineures) [16], et 20 à $25 \mathrm{p} .100$ de matières azotées non protéiques (acides aminés - urée - ammoniaque - bases azotées - créatine - nucléotides) [10].

Les protéines du lactosérum sont des protéines de haute qualité pour l'homme, car elles contiennent tous les acides aminés essentiels [38].

Le lactosérum est un produit instable ; en effet, les protéines solubles sont facilement dénaturées par la chaleur et deviennent insolubles : elles réagissent alors avec le lactose et la réaction Maillard s'établit. La protéolyse engendrée par l'action de la présure et des micro-organismes peut être mise en évidence par la détermination de l'azote soluble dans l'acide trichloracétique à 2 et 12 p. 100 . L'étude des acides aminés libres et de quelques produits de leur dégradation comme l'urée, l'ammoniaque, l'histamine et la tyramine permet de connaître les modifications éventuelles des lactosérums au cours de leur conservation.

\section{A. Teneur en insoluble}

Le lactosérum liquide contient des matières insolubles qui peuvent être déterminées quantitativement par centrifugation. Ce test consiste à évaluer pondéralement la dénaturation des protéines du sérum qui, selon Alais [1] sont sensibles au $\mathrm{pH}$, à la force ionique et à la température du milieu. Le tableau 3 montre que le pourcentage

TABLEAU 3

Taux d'insoluble dans les lactosérums acide et doux (g p. $100 \mathrm{~g} \mathrm{MS}$ )

\begin{tabular}{c|c|c}
\hline Conservation & Lactosérum acide & Lactosérum doux \\
\cline { 2 - 3 } Dès l'obtention & 1,33 & 0,44 \\
Après $5 \mathrm{j}$ à $+4^{\circ} \mathrm{C}$ & 2,06 & 0,56 \\
Après $5 \mathrm{j}$ à $+10^{\circ} \mathrm{C}$ & 2,08 & 1,12 \\
Après $5 \mathrm{j}$ à $+23^{\circ} \mathrm{C}$ & 2,64 & 2,12 \\
\hline
\end{tabular}


d'insoluble dans les lactosérums acides originels est nettement plus important que dans les lactosérums doux, la $\beta$-lactoglobuline étant précipitée. Après conservation de $5 \mathrm{j}$ à $+4^{\circ} \mathrm{C},+10^{\circ} \mathrm{C}$ et $+23^{\circ} \mathrm{C}$, le taux d'insoluble augmente dans les deux types de sérums pour atteindre des valeurs voisines.

\section{B. Teneur en hydroxyméthylfurfural}

Les répercussions des traitements de conservation auxquels sont soumis les différents lactosérums, sur l'utilisation digestive des protéines et sur leur utilisation métabolique sont à envisager également [9]. La valeur biologique d'une protéine dépend plus de la teneur en lysine disponible que totale. Le blocage de cet acide aminé réside principalement dans le fait que, par son radical $\alpha$-aminé, il est très sensible à la présence de sucres réducteurs dans le milieu [52]. La dégradation non enzymatique des sucres réducteurs montre que la réaction de Maillard est précédée de la formation d'un composé furfurique : l'hydroxyméthylfurfural [35].

Selon Keeney et Bassette [23], Samuelsson et Nielsen [45], Craig et al. [11], Della Monica et al. [12], l'hydroxyméthylfurfural (HMF) formé peut être déterminé quantitativement en présence d'acide 2 thiobarbiturique, par spectrophotométrie. L'absorption du composé formé est proportionnelle à la concentration en HMF.

Dans les lactosérums analysés dès leur obtention (tab. 4), les teneurs en HMF libre et potentiel sont sensiblement identiques. Ces

\section{TABLEAU 4}

Teneurs en HMF libre et potentiel des lactosérums acide et doux $\left(10^{-8}\right.$ Moles p. $\left.100 \mathrm{~g} \mathrm{MS}\right)$

\begin{tabular}{l|c|c}
\hline \multicolumn{1}{c|}{ Conservation } & HMF libre & HMF potentiel \\
\cline { 3 - 3 } & & \\
Lactosérum acide & & \\
Dès l'obtention & 2,49 & 7,82 \\
Après 5 j à + $4^{\circ} \mathrm{C}$ & 2,74 & 8,96 \\
Après 5 j à $+10^{\circ} \mathrm{C}$ & 2,27 & 9,3 \\
Après 5 j à $+23^{\circ} \mathrm{C}$ & 2,77 & 9,7 \\
Lactosérum doux & & \\
Dès l'obtention & 2,27 & 8,5 \\
Après 5 j à $+4^{\circ} \mathrm{C}$ & 2,15 & 10,3 \\
Après 5 j à $+10^{\circ} \mathrm{C}$ & 2,17 & 10,3 \\
Après 5 j à $+23^{\circ} \mathrm{C}$ & 2,18 & 11,3 \\
& & \\
\hline
\end{tabular}


teneurs augmentent mais dans de faibles proportions dans les lactosérums conservés à $+23^{\circ} \mathrm{C}$. Les quantités de HMF libre et potentiel sont mises en évidence au cours de l'analyse pour l'acidification à froid et à chaud de l'échantillon.

Ni l'origine des lactosérums, ni leur état de conservation ne peuvent être appréciés par ce test.

\section{Les différentes fractions azotées}

Afin de déterminer dans quelle mesure la technique fromagère utilisée entraîne des modifications de la fraction protidique, nous avons étudié sur les lactosérums originels et après conservation à $+4^{\circ} \mathrm{C}$ et $+23^{\circ} \mathrm{C}$, la teneur en azote total, en protéines totales, en peptides et polypeptides, en azote caséinique et protéines dénaturées du sérum.

L'azote est dosé, après minéralisation Kjeldahl, par une méthode colorimétrique adaptée à l'autoanalyseur Technicon [2, 14].

L'azote total du lactosérum se compose d'azote non caséinique, d'azote caséinique et des protéines dénaturées du sérum [31].

La fraction azotée non caséinique renferme la $\beta$-lactoglobuline, l' $\alpha$-lactalbumine, le sérum albumine, les globulines et les protéoses peptones, l'azote des glycopeptides et l'azote non protéique [55].

L'azote soluble dans l'acide trichloracétique à 12 p. 100 correspond à l'azote non protéique et à l'azote des glycopeptides. L'azote soluble dans l'acide trichloracétique à 2 p. 100 correspond à l'azote non protéique, à l'azote des glycopeptides et à l'azote des peptides $[1,54]$.

Dans le lactosérum acide (fig. 1), la teneur en azote total est supérieure à celle du lactosérum doux, bien que le taux des protéines soit plus bas. D'autre part, la fraction d'azote non protéique est plus importante, ce qui laisse supposer que dans le lactosérum acide originel, le degré de protéolyse est plus prononcé. Ceci sera vérifié ultérieurement en étudiant d'autres paramètres.

Le maintien à basse température des sérums apporte peu de modifications à ces résultats. Par contre à température ambiante, la teneur en protéines totales diminue, et celle de l'azote non protéique augmente. Une protéolyse microbienne des protéines, des peptides et des polypeptides se traduit par la libération de produits de dégradation qui peuvent être entre autres des acides aminés.

\section{Teneur en acides aminés libres}

La séparation et la détermination quantitative des acides aminés selon la méthode de Stein et Moore [48] permettent de suivre l'évolution du pool des acides aminés dans les lactosérums acide et doux. 


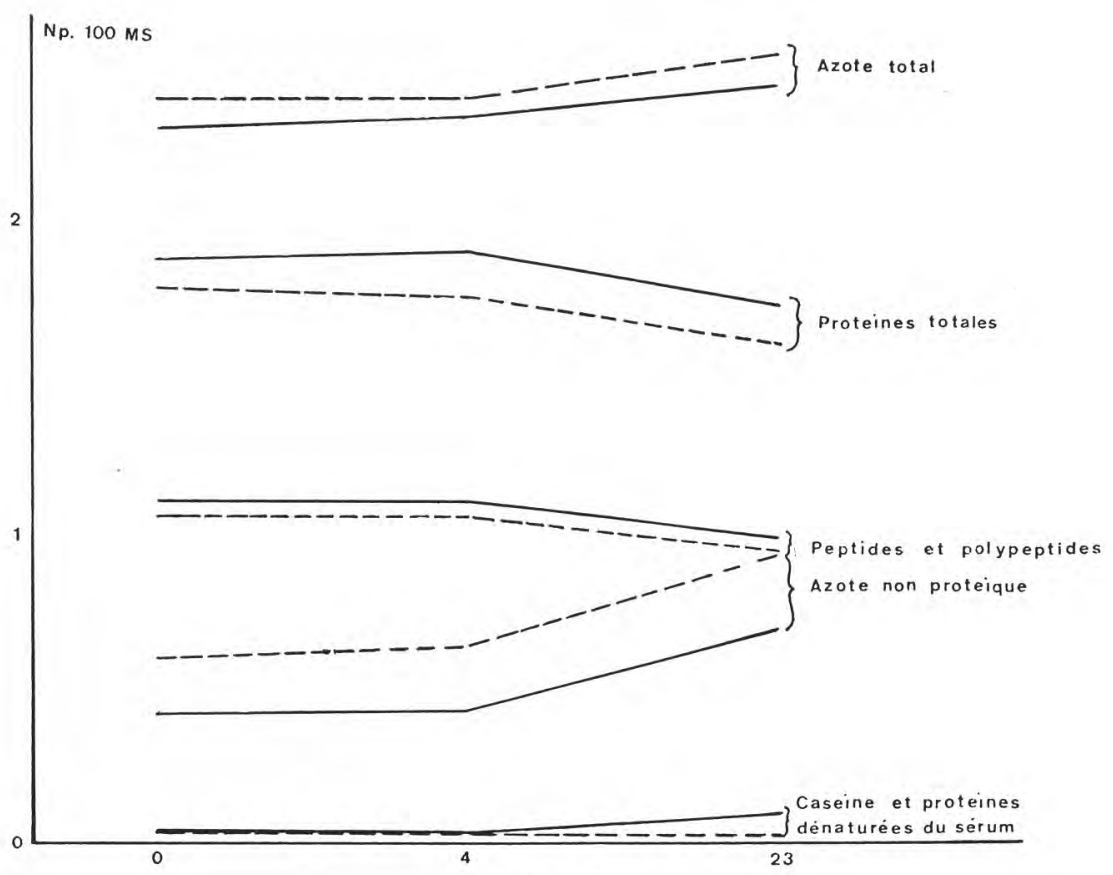

fig. 1

Composés azotés des lactosérums acide et doux

___ lactosérum acide, lactosérum doux.

0 : lactosérums analysés dès l'obtention.

4: lactosérums analysés après $5 \mathrm{j}$ de conservation à $+4^{\circ} \mathrm{C}$.

23 : lactosérums analysés après $5 \mathrm{j}$ de conservation à $+23^{\circ} \mathrm{C}$.

Les échantillons sont déprotéinisés par le procédé des fibres creuses. La solution dialysée obtenue est ajustée à un $\mathrm{pH}$ voisin de 2 avec l'acide chlorhydrique. La séparation des acides aminés est réalisée par passage sur une colonne de résine " chromobeads " type B, de $6 \mathrm{~mm}$ de diamètre et de $140 \mathrm{~cm}$ de long. L'élution d'une durée de $21 \mathrm{~h}$ est obtenue avec un gradient de tampon citrate réalisé en remplissant les neuf chambres de l'autograde par des tampons de $\mathrm{pH} 2,875$ et $\mathrm{pH} 4,74$ avec un débit d'élution fixé à $30 \mathrm{ml} / \mathrm{h}$. Les acides aminés sont dosés après coloration par la ninhydrine $[3,13$, $32,43]$.

\section{Remarques préliminaires}

Une résolution suffisamment bonne des composés phosphorés (phosphosérine, glycérophosphoéthanolamine, phosphoéthanolamine) 
TABLEAU 5

Taux moyen des acides aminés libres dans le lait de vache, et dans les lactosérums acide et doux frais

\begin{tabular}{|c|c|c|c|c|}
\hline \multirow{2}{*}{ Acides aminés } & \multicolumn{2}{|c|}{ Lait de vache (mg p. $100 \mathrm{ml}$ ) } & \multirow{2}{*}{$\begin{array}{c}\text { Lactosérum } \\
\text { acide } \\
\text { (mg p. } 100 \mathrm{ml})\end{array}$} & \multirow{2}{*}{$\begin{array}{c}\text { Lactosérum } \\
\text { doux } \\
(\mathrm{mg} \mathrm{p.} 100 \mathrm{ml})\end{array}$} \\
\hline & $\begin{array}{c}\text { RAZEMON } \\
\text { PINTA (1971) }\end{array}$ & $\begin{array}{l}\text { GHADIMI } \\
\text { (1963) }\end{array}$ & & \\
\hline Taurine & 0,32 & 0,61 & 0,99 & 0,94 \\
\hline Méthionine sulfoxyde & 0,34 & - & 0,14 & 0,11 \\
\hline Acide aspartique & 0,25 & 0,18 & 1,35 & 0,08 \\
\hline Thréonine & 0,01 & 0,09 & 0,10 & 0,05 \\
\hline Asparagine + Glutamine* & 0,04 & 0,33 & 0,23 & traces \\
\hline Sérine & 0,02 & 0,08 & 0,14 & 0,07 \\
\hline Acide glutamique & 1,44 & 1,77 & 9,08 & 1,77 \\
\hline Citrulline & 0,04 & traces & 0,42 & 0 \\
\hline Glycine & 0,71 & 0,59 & 0,04 & 0,42 \\
\hline Alanine & 0,24 & 0,26 & 0,19 & 0,20 \\
\hline$\alpha$-aminobutyrique & 0,03 & 0,03 & traces & 0 \\
\hline Valine & 0,09 & 0,21 & 0,27 & 0,09 \\
\hline Cystine & - & 0,05 & 0 & 0 \\
\hline Méthionine & 0,03 & traces & 0,04 & 0 \\
\hline Isoleucine & 0,02 & 0,06 & 0,05 & traces \\
\hline Leucine & 0,02 & 0,13 & 0,28 & 0,05 \\
\hline Tyrosine & 0,03 & 0,08 & 1,40 & 0,07 \\
\hline Phénylalanine & 0,02 & 0,10 & 0,56 & 0,06 \\
\hline$\beta$-aminoisobutyrique & - & - & 0 & 0 \\
\hline$\gamma$-aminobutyrique & & 0,61 & 0,06 & 0 \\
\hline Ethanolamine & & - & 0,32 & traces \\
\hline Ornithine & & - & 0,67 & 0,08 \\
\hline Lysine & & 0,42 & 2,98 & 0,17 \\
\hline Histidine & & 0,10 & 1,11 & 0,13 \\
\hline Arginine & & 0,21 & 1,26 & 0,13 \\
\hline Total & 7,78 & 6,11 & 21,7 & 4,59 \\
\hline
\end{tabular}

* Asparagine + Glutamine sont calculées en glutamine. 


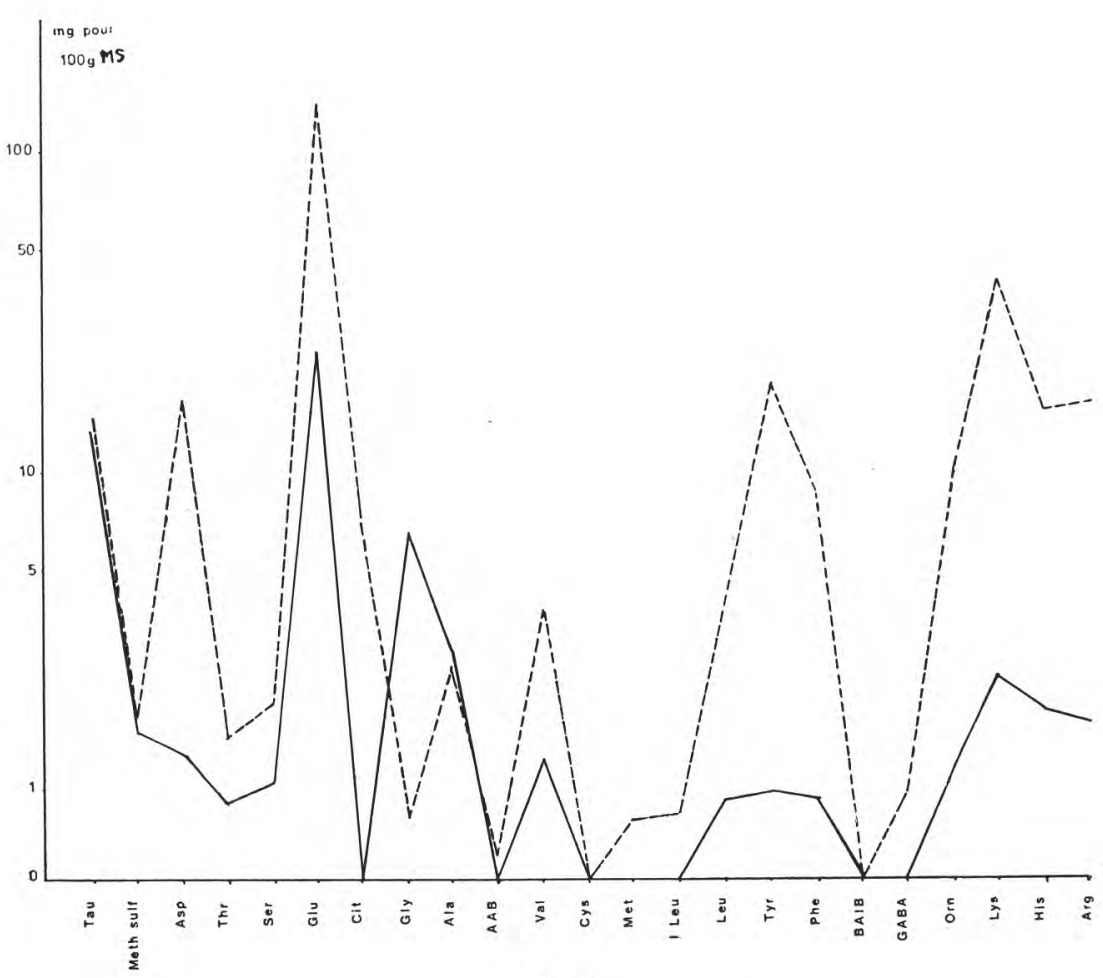

fig. 2

Acides aminés libres des lactosérums originels

—— lactosérum acide, — lactosérum doux

et de l'acide cystéique n'a pu être obtenue pour permettre une interprétation quantitative. Toutefois, dans les lactosérums doux, la concentration de ces composés reste toujours inférieure à celle observée dans les lactosérums acides.

Deux pics n'ont pu être identifiés. Le premier sort immédiatement après la méthionine sulfoxyde. Sa concentration ne varie pratiquement pas dans le lactosérum acide au cours des différents essais. Absent dans le lactosérum doux originel, il apparaît dans les sérums maintenus $5 \mathrm{j}$ à $+23^{\circ} \mathrm{C}$. Le deuxième pic non identifié figure entre la citrulline et la glycine, seulement sur les chromatogrammes des lactosérums acide et doux conservés $5 \mathrm{j}$ à $+23^{\circ} \mathrm{C}$.

L'équipement dont nous disposons ne nous a pas permis de doser la proline et l'hydroxyproline. Nous n'avons pas retenu les résultats 
trouvés pour l'ammoniaque et l'urée. Si les pics sont toujours très nets, les taux trouvés sont extrêmement variables et ne peuvent pas toujours être évalués à partir des dilutions utilisées pour le dosage des acides aminés.

\section{Composition des lactosérums analysés dès leur obtention}

Dans le tableau 5, figure la composition moyenne en acides aminés libres des lactosérums acide et doux ainsi que celle du lait $[17,41]$.

Par comparaison avec l'ensemble des acides aminés libres du lait [17], on note dans le lactosérum acide la disparition de la cystine, une faible teneur en glycine et en acide $\gamma$-aminobutyrique, une augmentation du pourcentage de taurine, d'acide aspartique, d'acide glutamique, de citrulline, de tyrosine, de phénylanine, de lysine, d'histidine et d'arginine. En revanche, la concentration des autres acides aminés n'est pas très différente.

Le lactosérum de pâte cuite, par rapport au lactosérum de pâte fraîche, est pauvre en acides aminés. Nous remarquons l'absence de cystine, de méthionine, de citrulline, d'acide $\gamma$-aminobutyrique, d'acide $\alpha$-aminobutyrique et d'acide $\beta$-aminoisobutyrique.

Il ressort de cette étude que pour chacun des deux types de lactosérum examinés à l'état frais, il est possible d'établir « un profil chromatographique » caractéristique (fig. 2).

Les lactosérums de pâte cuite ou de pâte fraîche, dès l'origine, ne referment ni cystine, ni acide $\beta$-aminoisobutyrique. sérum

3. Evolution du pool des acides aminés dans les deux types de

La concentration du pool des acides aminés du lactosérum acide augmente en fonction de la température de conservation. Dans le lactosérum de pâte cuite, la teneur globale des acides aminés diminue après $5 \mathrm{j}$ de conservation à $+4^{\circ} \mathrm{C}$ mais augmente très rapidement à $+23^{\circ} \mathrm{C}$ (fig. 3).

Cette étude met en relief :

- la grande différence de concentration des acides aminés totaux selon les deux types de sérum, le plus riche étant celui du lactosérum acide ;

- l'augmentation progressive mais relativement faible de l'ensemble des acides aminés dans le lactosérum acide au cours de la conservation à basse température $\left(+4^{\circ} \mathrm{C}\right)$ et à température ambiante $\left(+23^{\circ} \mathrm{C}\right)$;

- la légère récession du taux global des acides aminés lors de la conservation à $+4^{\circ} \mathrm{C}$ dans le lactosérum doux, alors qu'à $+23^{\circ} \mathrm{C}$ il présente un accroissement très important. 


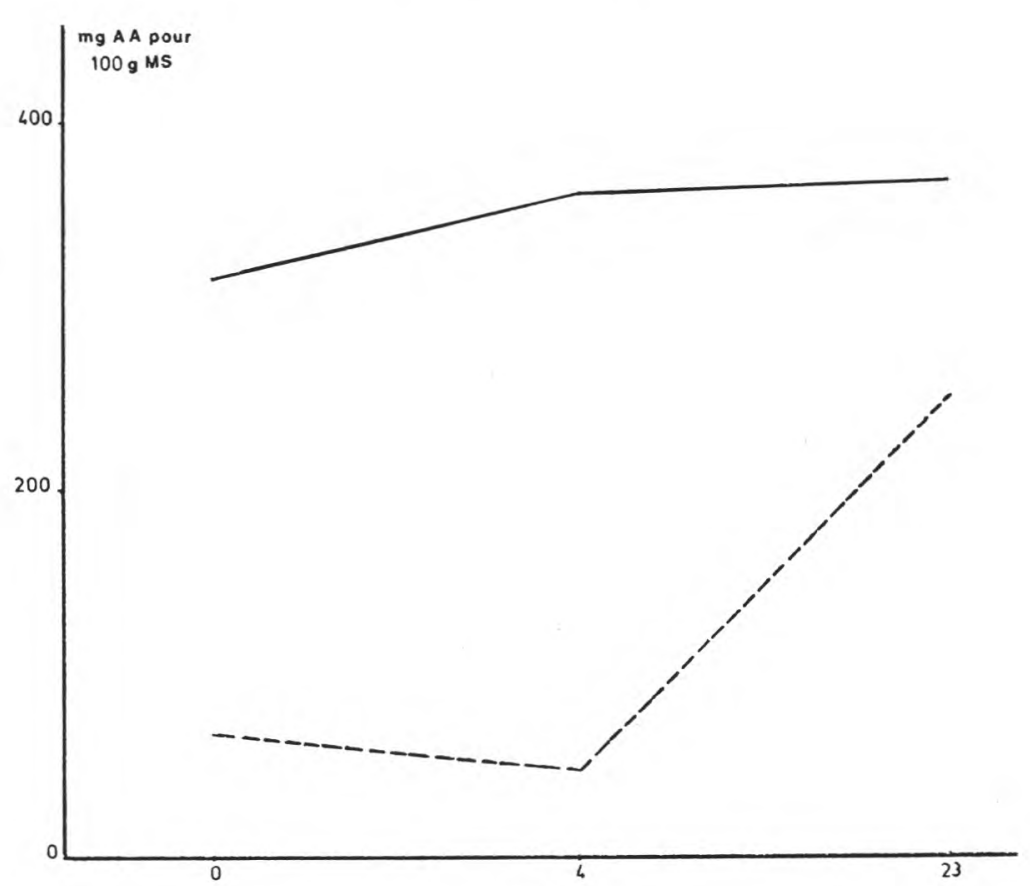

fig. 3

Evolution du total des acides aminés libres lactosérum acide, lactosérum doux

0 : lactosérums analysés dès l'obtention,

4: lactosérums analysés après $5 \mathrm{j}$ de conservation à $4^{\circ} \mathrm{C}$,

23: lactosérums analysés après $5 \mathrm{j}$ de conservation à $23^{\circ} \mathrm{C}$.

La composition de l'ensemble des acides aminés libres dans le sérum frais et dans le sérum maintenu $5 \mathrm{j}$ à $+4^{\circ} \mathrm{C}$ et $+23^{\circ} \mathrm{C}$ figure dans le tableau 6.

a) Après un séjour de $5 \mathrm{j}$ à $+4^{\circ} \mathrm{C}$, les acides aminés libres du point de vue qualitatif, ne varient pas sauf l'isoleucine qui disparaît du lactosérum doux. Du point de vue quantitatif, les faibles variations enregistrées pour chaque acide aminé ne modifient guère « le profil chromatographique » de chacun des deux types de sérum.

b) Après un séjour de $5 \mathrm{j}$ à $+23^{\circ} \mathrm{C}$, les deux types de lactosérum présentent des pools d'acides aminés très différents de ceux obtenus à l'analyse des lactosérums frais (fig. 4).

Comme pour les acides aminés libres de fromage [28], le profil des courbes traduit un ensemble extrêmement complexe de dégrada- 
TABLEAU 6. - Acides aminés libres dans le lactosérum acide et doux (mg p. $100 \mathrm{~g}$ MS)

\begin{tabular}{|c|c|c|c|c|c|c|}
\hline \multirow{3}{*}{ Acides aminés } & \multicolumn{3}{|c|}{ Lactosérum acide } & \multicolumn{3}{|c|}{ Lactosérum doux } \\
\hline & \multirow{2}{*}{ originel } & \multicolumn{2}{|c|}{ conservé $5 \mathrm{j}$} & \multirow{2}{*}{ originel } & \multicolumn{2}{|c|}{ conservé $5 \mathrm{j}$} \\
\hline & & $+4^{\circ} \mathrm{C}$ & $+23^{\circ} \mathrm{C}$ & & $+4^{\circ} \mathrm{C}$ & $+23^{\circ} \mathrm{C}$ \\
\hline Taurine & 14,69 & 16,4 & 15,2 & 14,2 & 13,9 & 15.0 \\
\hline Méthionine sulfoxyde & 2,04 & 2,11 & 2,86 & 1,62 & 1,52 & $\begin{array}{r}1 J, 0 \\
2,32\end{array}$ \\
\hline Acide aspartique & 19,7 & 21,8 & 20,6 & 1,24 & 1,60 & 2,7 \\
\hline Thréonine & 1,48 & 1,18 & 5,7 & 0,75 & 0,22 & 16,6 \\
\hline Asparagine + Glutamine* & 3,42 & 2,22 & 6,8 & traces & traces & 5,2 \\
\hline Sérine & 2,05 & 1,84 & 6,4 & 1,02 & traces & 5,7 \\
\hline Acide glutamique & 133 & 150,4 & 123 & 26,7 & 17,2 & 14,4 \\
\hline Citrulline & 6,15 & 6,89 & 5,3 & 0 & 0 & 3,6 \\
\hline Glycine & 0,55 & 0,83 & 1,30 & 6,4 & 5,0 & 10,9 \\
\hline Alanine & 2,79 & 4,31 & 13,6 & 3,10 & 2,46 & 21,8 \\
\hline$\alpha$-aminobutyrique & traces & traces & 1,43 & 0 & 0 & 1,16 \\
\hline Valine & 3,95 & 4,87 & 17,4 & 1,22 & 0,59 & 16,1 \\
\hline Cystine & 0 & 0 & 1,28 & 0 & 0 & 2,9 \\
\hline Méthionine & 0,53 & 0,31 & 5,5 & 0 & 0 & 0,52 \\
\hline Isoleucine & 0,60 & 0,38 & 6,45 & traces & 0 & 7,3 \\
\hline Leucine & 4,10 & 4,71 & 20,6 & 0,80 & traces & 6,5 \\
\hline Tyrosine & 20,5 & 27,5 & 22,8 & 0,94 & 0,45 & 2,4 \\
\hline Phénylalanine & 8,3 & 8,78 & 12,3 & 0,80 & 0,50 & 2,10 \\
\hline$\beta$-aminoisobutyrique & 0 & 0 & 6,6 & 0 & 0 & 33 \\
\hline$\gamma$-aminobutyrique & 0,94 & traces & 6,9 & 0 & 0 & 59 \\
\hline Ethanolamine & 4,7 & 5,5 & 10,7 & traces & traces & 4,1 \\
\hline Ornithine & 9,9 & 12,2 & 12,7 & 1,14 & 1,37 & 4,5 \\
\hline Lysine & 43,2 & 44,9 & 27,9 & 2,63 & 2,8 & 9,0 \\
\hline Histidine & 16,3 & 20,0 & 11,8 & 2,02 & 1,43 & 5,4 \\
\hline Arginine & 18,2 & 24 & 4,4 & 1,8 & traces & 1,27 \\
\hline Total & 317,2 & 361,3 & 369,5 & 66,7 & 49,5 & 253,5 \\
\hline
\end{tabular}

* Asparagine + Glutamine sont calculées en glutamine. 


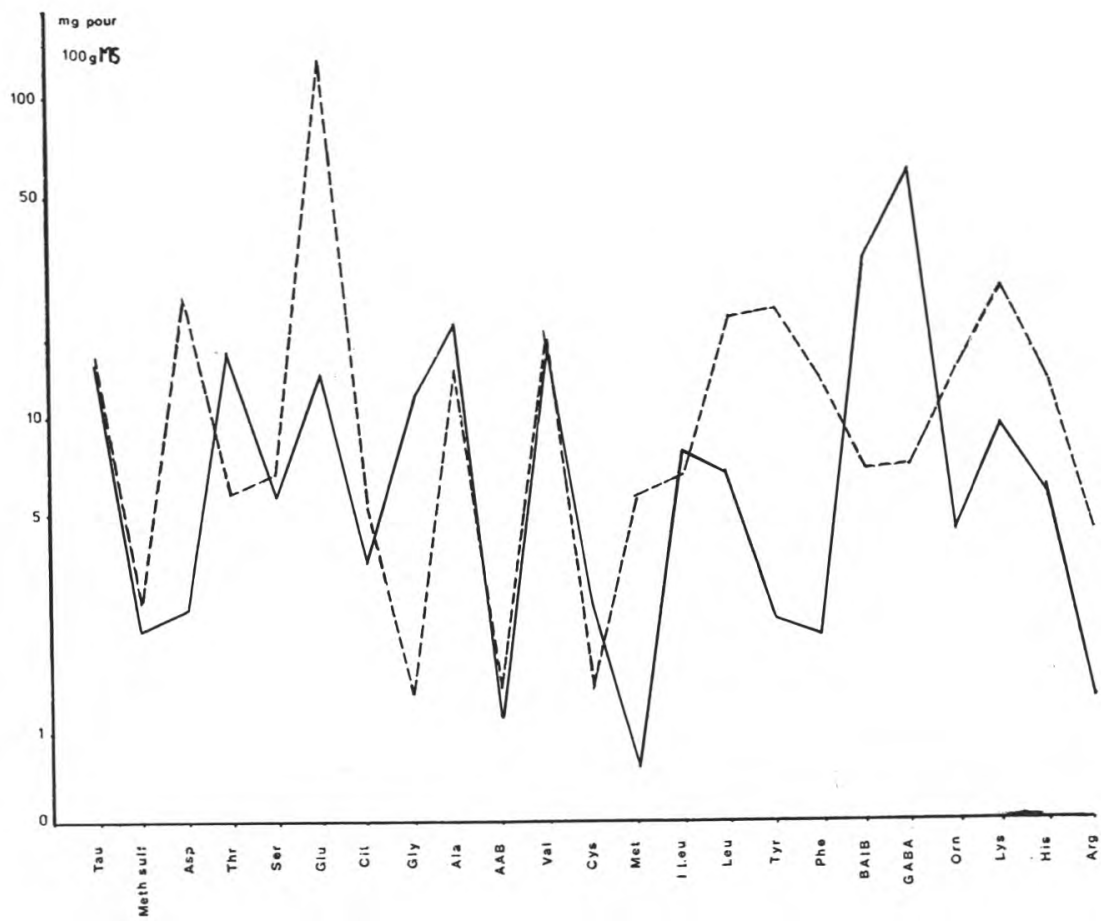

fig. 4

Acides aminés libres des lactosérums c ınservés $5 \mathrm{j}$ à température ambiante $\left(+23^{\circ} \mathrm{C}\right)$

- lactosérum acide, lactosérum doux

tions et de synthèses. Des acides aminés non présents dans le sérum frais se forment par décarboxylation, par désamination oxydative ou par hydrolyse des acides aminés originels, exemple :

- l'acide $\alpha$-aminobutyrique à partir de la méthionine ;

- l'acide $\gamma$-aminobutyrique à partir de l'acide glutamique ;

- l'ornithine et la citrulline aux dépens de l'arginine [25].

Les proportions relatives de chacun peuvent être notablement modifiées du fait de remaniements ou de dégradations enzymatiques secondaires.

La cystine, acide aminé absent dans les deux types de sérum originel, apparaît au cours de la conservation à un taux plus élevé dans le lactosérum doux que dans le lactosérum acide. La dénatura- 
tion de la $\beta$-lactoglobuline suivie de la protéolyse, libère de la cystine. La présence de présure dans le lactosérum doux pourrait expliquer l'augmentation de cet acide aminé.

Quelques acides aminés comme l'arginine et l'acide glutamique tendent à disparaître dans les deux types de sérum. De plus dans le lactosérum acide, une diminution de la teneur en lysine et en histidine est enregistrée. Des réactions de désamination, d'hydrolyse ou d'oxydation peuvent être responsables de ces modifications.

Certains acides aminés sont présents à des taux plus élevés dans les lactosérums maintenus à température ambiante. C'est le cas de la thréonine, de la sérine, de l'alanine, de la valine, de l'isoleucine et de la leucine. Ces différences peuvent s'expliquer par une libération préférentielle lors de la protéolyse. Il pourrait en être ainsi pour la leucine et l'isoleucine qui appartiennent aux parties terminales des molécules de la $\beta$-lactoglobuline et de l' $\alpha$-lactalbumine $[29,31]$. La présence de ces deux acides aminés serait l'indice d'une activité carboxy et aminopeptidasique particulièrement grande. Le taux élevé d'un acide aminé peut aussi s'expliquer par une production due à une excrétion ou à une lyse microbienne, tel pourrait être le cas de l'alanine que l'on sait présente à un fort pourcentage dans les protéines de certains micro-organismes.

Nous avons relevé la présence non systématique de la $\beta$-alanine qui serait libérée en même temps que l'acide $\beta$-aminoisobutyrique lors de la dégradation des bases pyrimidiques [21].

Contrairement à l'ensemble des acides aminés, la thréonine, la glycine, l'acide $\beta$-aminoisobutyrique et l'acide $\gamma$-aminobutyrique sont présents à des taux plus élevés dans le lactosérum de pâte cuite conservé à $+23^{\circ} \mathrm{C}$ que dans celui de pâte fraîche. L'activité protéasique de la présure semble intensifier la protéolyse au cours de la conservation à température ambiante du lactosérum doux.

\section{Interprétation}

Il semble donc que le « profil chromatographique » des acides aminés d'un type de sérum donné soit le reflet des activités enzymatiques qui se manifestent au cours de la conservation. Les sérums acides sont plus riches en acides aminés libres que les lactosérums doux, quel que soit le stade de l'étude. Cependant, la protéolyse dans les sérums de pâte cuite conservés à température ambiante est très intense. La teneur en acides aminés libres, conséquence de l'altération des protéines, se trouve triplée par rapport à la teneur originelle. La présence d'acide $\beta$-aminoisobutyrique dans les lactosérums acide et doux, conservés à température ambiante, témoigne d'une activité protéolytique et d'une fermentation butyrique. Dans les lactosérums originels, nous n'avons pas trouvé de teneurs élevées en acides butyriques aminés. 
D'après notre étude, l'acide $\beta$-aminoisobutyrique indique l'existence de processus de dégradations protéiques et glucidiques dans les lactosérums liquides.

Dans ce qui précède ont été étudiées les modifications des protides en fonction de la technique fromagère employée et des conditions de conservation appliquées (insoluble, protéines-lactose, azote). L'étude des acides aminés libres a montré l'évolution des processus de dégradations et de synthèses protidiques au sein des lactosérums liquides. Il paraît intéressant de suivre la dégradation des acides aminés en étudiant quelques composés issus de leur désamination et de leur décarboxylation : urée et ammoniaque, histamine et tyramine qui sont des monoamines ayant la propriété d'être des hormones tissulaires à action locale.

\section{E. Teneur en ammoniaque}

L'ammoniaque, comme l'urée, est un composé libéré lors des réactions de désamination des acides aminés. En pratique, un lactosérum frais ne doit pas renfermer de substances ammoniacales. Mais les résultats publiés par Février, Shahani et Sommer, Kieferle et Gloetzl [10], montrent de très grandes divergences pour un même type de lactosérum (tab. 7).

\section{TABLEAU 7}

Teneur en azote ammoniacal de quelques lactosérums (mg p. 100)

\begin{tabular}{|c|c|c|c|}
\hline & $\begin{array}{l}\text { Février } \\
\text { (1971) }\end{array}$ & $\begin{array}{c}\text { SHAHANI, SOMMER } \\
(1950)\end{array}$ & $\begin{array}{l}\text { KIEFERLE, GLOETZL } \\
\text { (1931) }\end{array}$ \\
\hline Pâte fraîche & $11,8-15,5$ & 0,8 & 1,1 \\
\hline Emmental & $3,6-7,3$ & - & - \\
\hline Comté & 2,6 & - & - \\
\hline
\end{tabular}

Nous avons repris le protocole opératoire de RHO [42] pour déterminer quantitativement l'azote ammoniacal dans les échantillons.

Les lactosérums originels obtenus par acidification ou emprésurage du lait renferment d'assez faibles quantités d'ammoniaque. Ces teneurs (tab. 8) ne varient presque pas dans les lactosérums conservés $5 \mathrm{j}$ à $+4^{\circ} \mathrm{C}$ mais augmentent d'autant plus que la température de conservation est plus élevée. 
TABLEAU 8. - Teneurs en ammoniaque, urée, tyramine et histamine des lactosérums acide et doux

\begin{tabular}{|c|c|c|c|c|c|c|c|c|}
\hline \multirow{2}{*}{ Conservation } & \multicolumn{2}{|c|}{$\begin{array}{c}\text { Teneur en azote } \\
\text { ammoniacal } \\
\text { (mg N p. } 100 \mathrm{~g} \mathrm{MS} \text { ) }\end{array}$} & \multicolumn{2}{|c|}{$\begin{array}{c}\text { Teneur en azote } \\
\text { uréique } \\
\text { (mg N p. } 100 \text { g MS) }\end{array}$} & \multicolumn{2}{|c|}{$\begin{array}{c}\text { Teneur } \\
\text { en tyramine } \\
(\mathrm{mg} \text { p. } 100 \mathrm{~g} \mathrm{MS})\end{array}$} & \multicolumn{2}{|c|}{$\begin{array}{c}\text { Teneur } \\
\text { en histamine } \\
(\mathrm{mg} \text { p. } 100 \mathrm{~g} \mathrm{MS})\end{array}$} \\
\hline & acide & doux & acide & doux & acide & doux & acide & doux \\
\hline Dès l'obtention & 16 & 28 & 100 & 138 & 0,25 & 0,23 & 0,24 & 0,08 \\
\hline Après $5 \mathrm{j}$ à $+4^{\circ} \mathrm{C}$ & 18 & 43 & 90 & 82 & 0,27 & 0,23 & 0,26 & 0,09 \\
\hline Après $5 \mathrm{j}$ à $+10^{\circ} \mathrm{C}$ & 37 & 86 & 94 & 58 & 0,29 & 0,27 & 0,27 & 0,05 \\
\hline Après $5 \mathrm{j}$ à $+23^{\circ} \mathrm{C}$ & 81 & 120 & 56 & 23 & 0,50 & 0,55 & 0,51 & 0,14 \\
\hline
\end{tabular}




\section{F. Teneur en urée}

L'urée est un constituant normal du lait, a fortiori du lactosérum. D'après les valeurs trouvées dans la littérature (tab. 9) l'urée est l'un des constituants le plus abondant de la fraction azotée non protéique [10].

\section{TABLEAU 9}

Teneur en azote uréique de quelques lactosérums (mg p. 100)

\begin{tabular}{|c|c|c|c|}
\hline & $\begin{array}{l}\text { Février } \\
\text { (1971) }\end{array}$ & $\begin{array}{c}\text { SHAHANI, SOMMER } \\
(1950)\end{array}$ & $\begin{array}{l}\text { KIEFERLE, GLOETZL } \\
\text { (1931) }\end{array}$ \\
\hline Pâte fraîche & $6,1-8,4$ & 8,6 & 10,1 \\
\hline Emmental & $7,9-13,4$ & - & - \\
\hline Comté & 14,6 & - & - \\
\hline
\end{tabular}

L'urée a été déterminée selon le protocole opératoire de Mc Dowell $[7,30,53]$. Au cours de la conservation des lactosérums, la teneur en azote uréique diminue (tab. 8). Ceci serait dû à la présence de micro-organismes libérant de l'uréase ; celle-ci hydrolyse l'urée en ammoniaque et en gaz carbonique [20]. Le développement de la flore microbienne étant plus important dans les lactosérums conservés à $+10^{\circ} \mathrm{C}$ et $+23^{\circ} \mathrm{C}$, la concentration de l'uréase est plus forte, ce qui explique la diminution du taux d'azote uréique.

Ainsi, dans l'augmentation de la teneur en azote ammoniacal une forte proportion provient de l'hydrolyse de l'urée.

\section{G. Teneur en tyramine}

La présence de tyramine et d'histamine dans les aliments témoigne de l'activité d'un processus fermentaire. La tyramine dérive de la tyrosine par décarboxylation sous l'action de bactéries de putréfaction [20]. Price et Smith [40], Kayaalp et al. [22], Bonnet et Nepveux [6] ont été amenés à étudier le rôle physiopathologique de la tyramine, sympathomimétique puissant, en particulier sur le système cardio-vasculaire. Certains fromages sont assez riches en tyramine, les valeurs trouvées dans la littérature sont rassemblées dans le tableau 10. 
TABLEAU 10

Teneur en tyramine de quelques fromages (mg p. $100 \mathrm{~g}$ )

\begin{tabular}{l|c|c|c}
\hline & Bonnet (1970) & Price (1971) & Kayalip (1970) \\
\cline { 2 - 3 } Camembert & 8,0 & - & - \\
Brie & 18,0 & - & - \\
Emmental & 22,5 & - & - \\
Gruyère & 51,6 & 1,1 à 118,4 & 6,43 \\
Caséine & 0,45 & - & - \\
Fromage blanc & - & - & 1,75 \\
\end{tabular}

Les fromages à pâte cuite renferment de la tyramine en quantité appréciable. Il nous a paru intéressant de savoir si les lactosérums liquides contenaient cette amine, de connaitre sa concentration et son devenir au cours de la conservation à différentes températures.

Il existe une méthode colorimétrique de dosage basée sur la réaction de la tyrosine avec le nitrosonaphtol. Waalkes et Udenfriend ont montré que le composé obtenu était hautement fluorescent $[33,36,51]$. Dérivé décarboxylé de la tyrosine, la tyramine possède les mêmes caractéristiques de fluorescence. Cependant, son caractère basique lui permet d'être séparée par extraction à l'aide d'un solvant $[22,34,49]$.

Les deux types de lactosérum examinés dès leur obtention (tab. 8), renferment une assez faible quantité de tyramine (acide 0,255 - doux $0,230 \mathrm{mg}$ p. $100 \mathrm{~g}$ MS). Cette teneur n'évolue pratiquement pas dans les lactosérums conservés à $+4^{\circ} \mathrm{C}$ et $+10^{\circ} \mathrm{C}$ mais augmente dans les lactosérums conservés à température ambiante.

L'augmentation de la teneur en tyramine est probablement due à l'accroissement de la population bactérienne et principalement des bactéries putréfiantes. D'après les travaux de Price [40], les lactobacilles introduits dans la fabrication des fromages ne renferment pas cette enzyme. Les agents responsables de la décarboxylation de la tyrosine seraient des bactéries aérobies apportées par le lait cru ou par les souches de levains mésophiles. Ils peuvent être aussi des bactéries de contamination. Malgré cette augmentation au cours de la conservation à température ambiante, les teneurs en tyramine sont 
nettement inférieures à celles trouvées dans les fromages [6, 22]. Les lactosérums même altérés, ne contiennent pas une quantité de tyramine suffisamment importante pour limiter leur emploi en alimentation.

\section{H. Teneur en histamine}

L'histamine est le témoin d'une protéolyse dépassant le stade des acides aminés libres. Elle se forme par décarboxylation de l'histidine [19]. Alors que l'histamine est peu souhaitable voire toxique, l'histidine est indispensable car elle ne peut être synthétisée par l'organisme [37].

Le dosage est basé sur la fluorimétrie du dérivé de condensation de l'histamine avec l'ortho-phtaldéhyde [27, 33, 51], après extraction par le butanol en milieu acide [4].

Depuis la publication de Shore [47], les possibilités d'interférence de la méthode fluorimétrique ont été étudiées et répertoriées par de nombreux auteurs, en particulier par Carlini et Green [8], et Kremzner et Pfeiffer [26]. Pour ces auteurs, l'histidine, l'arginine, l'agmatine (arginine décarboxylée), l'ammoniaque peuvent donner une fluorescence avec l'orthophtaldéhyde. Ces corps sauf l'ammoniaque, sont présents dans les lactosérums à des teneurs plus faibles que les seuils proposés par Ruff et al. [44]. Pour l'ammoniaque, Plumas [37] préconise une extraction, en fin de réaction, par addition d'alcool isoamylique quand le taux est supérieur à $4 \mathrm{mg} / \mathrm{ml}$.

A l'origine (tab. 8), les deux types de lactosérum ont des taux d'histamine nettement différents. Les ferments lactiques introduits dans le lait cru pour obtenir un caillé acide et, subséquemment le lactosérum, doivent renfermer l'enzyme de décarboxylation de l'histidine. Le pH acide 4,5-4,6 du sérum active l'histidine décarboxylase [21] d'où la teneur élevée en cette amine du lactosérum de pâte fraîche. La conservation pendant $5 \mathrm{j}$ à $+4^{\circ} \mathrm{C}$ et $+10^{\circ} \mathrm{C}$, n'entraîne pas de différences notables de concentration. A la température de $+23^{\circ} \mathrm{C}$, une nette augmentation est enregistrée qui laisse supposer une prolifération intense des micro-organismes contenant l'histidine décarboxylase. Dans le lactosérum de pâte cuite, les teneurs en histamine sont beaucoup plus faibles et restent toujours inférieures à la teneur minimale du lactosérum acide. La connaissance de la flore microbienne du lait cru mis en ouvre permettrait une meilleure interprétation de ces résultats.

\section{CONCLUSION}

Les possibilités de valorisation des lactosérums semblent très importantes. Les nombreux produits dérivés obtenus et les recherches 
effectuées sur le sérum, au stade pilote dans diverses entreprises, en témoignent.

La valeur nutritionnelle du sérum peut conduire ce produit à trouver de plus en plus d'applications dans le domaine de l'alimentation humaine comme substitut du lait écrémé, comme adjuvant dans de nombreux produits alimentaires déshydratés, comme source de protéines, comme source de lactose. La nécessité de développer les nouvelles techniques de traitement est impérative afin que les utilisateurs aient à leur disposition des produits de qualité.

L'origine des lactosérums liquides, analysés dès l'obtention, peut être révélée par l'étude de quelques critères chimiques tels l'acidité, les matières minérales, les acides aminés libres. En effet, le lactosérum de pâte fraîche ou acide renferme une quantité d'acide lactique nettement supérieure à celle du lactosérum de pâte cuite ou doux. L'activité protéolytique des ferments lactiques commence dès l'égouttage du caillé de pâte fraîche en libérant des acides aminés. Par contre, le lactosérum de pâte cuite, tout comme le lait, contient peu d'acides aminés libres.

L'analyse des lactosérums acide et doux maintenus $5 \mathrm{j}$ à basse température $\left(+4^{\circ} \mathrm{C}\right)$, ne montre aucune variation notable de la composition chimique de chacun d'eux. Le développement des fermentations est très limité dans le sérum doux et n'évolue pratiquement pas dans le lactosérum acide. Après un séjour à $+23^{\circ} \mathrm{C}$, les processus fermentaires et protéolytiques se développent. La composition chimique des lactosérums se modifie. L'effet principal est une diminution rapide de la teneur en matière sèche avec une acidification importante du lactosérum doux. Le lactose se transforme en acide lactique, en acides organiques et en composés volatils. Les traces d'acides $\alpha$ et $\gamma$-aminobutyrique, constatées dans le lactosérum de pâte fraîche originel sont peu souhaitées dans les aliments. Ces teneurs augmentent dans le sérum acide maintenu à $+23^{\circ} \mathrm{C}$ mais en proportions moindres que celles enregistrées pour le lactosérum de pâte cuite. Dans l'ensemble, par rapport à la composition des sérums originels, le lactosérum doux conservé à température ambiante présente une altération des protéines beaucoup plus marquée que le lactosérum acide.

Au cours du stockage des lactosérums liquides à température ambiante, deux acides aminés apparaissent : la cystine qui proviendrait de la dénaturation suivie d'une protéolyse des protéines et l'acide $\beta$-aminoisobutyrique dont la formation pourrait être due à un processus fermentaire. En effet la fermentation butyrique du lactose élabore de l'acide butyrique qui, par transamination, forme l'acide $\alpha$-aminobutyrique, l'acide $\gamma$-aminobutyrique et l'acide $\beta$-aminoisobutyrique.

L'acide $\beta$-aminoisobutyrique et l'ammoniaque peuvent être retenus comme "critère de qualité » du produit. Leur présence correspond à une altération par fermentation protéolytique. 
Ces faits montrent que les lactosérums liquides peuvent être conservés $5 \mathrm{j}$ à basse température sans qu'apparaissent de dégradations majeures ; lorsque la température s'élève, leur altération s'accélère. En effet, à température ambiante la dénaturation et la dégradation des protides font apparaître des composés indésirables parmi lesquels certaines amines considérées comme toxiques.

Ces conclusions ne sont applicables qu'aux lactosérums liquides. Le cas des lactosérums concentrés ou en poudre doit faire l'objet d'investigations particulières.

\section{Remerciements}

Nous tenons à exprimer nos remerciements et notre gratitude à M. Pantaléon, Mme Serres et Mme Amariglio pour leur aide et leurs conseils au cours de ce travail.

\section{Rés u m é}

L'analyse du lactosérum liquide frais et conservé pendant $5 \mathrm{j}$ à différentes températures $\left(+4^{\circ} \mathrm{C}\right.$ et $\left.+23^{\circ} \mathrm{C}\right)$ montre l'influence $\mathrm{du}$ mode d'obtention (lactosérum acide et lactosérum doux) et des conditions de stockage sur sa composition.

L'évolution de la dégradation des composés azotés est suivie par l'étude de l'insoluble, de la concentration en hydroxyméthylfurfural, des teneurs en protides, en acides aminés libres, en ammoniaque, en urée, en tyramine et en histamine.

La présence d'acides aminobutyriques et plus particulièrement de l'acide $\beta$-aminoisobutyrique, même en faible quantité, révèle la dégradation protéique et glucidique du lactosérum liquide.

\section{S u m m a r y}

The analysis of fresh liquid whey, or of five day old liquid whey stored at different temperatures $\left(4^{\circ} \mathrm{C}\right.$ and $23^{\circ} \mathrm{C}$ ) shows the influence of its preparation method (acide whey and swett whey) and of the storage conditions on its composition.

The evolution of the degradation of nitrogen fractions is followed by a study of the insoluble substances, of the concentration of hydroxymethylfurfural, of the amounts of free aminoacids, of ammonia, of urea, of tyramine and of histamine contained.

The presence of aminobutyric acids and particuliarly of $\beta$-aminoisobutyric acid, even in small amounts, reveals the degradation of the protides and glucides contained in the liquid whey. 


\section{Bibliographie}

[1] AlaIs (C.) (1965). - Science du lait.Principes des techniques laitières. $2^{\mathrm{e}}$ édition, sep.

[2] Amariglio (S.), Pondaven (A.) (1973). - Contribution à l'étude des méthodes d'analyse automatique du lait et du lactosérum secs. Le Lait, 527, 386-412.

[3] Technical manual, amino acid analyzer. - Technicon Instruments Corp., Ardsley, New York.

[4] Bio-science Laboratories, Beckman. - Fluorometric Procedures. Clinical Analysis for Histamine in Blood.

[5] BlaNC (B.) (1969). - Lactosérum et produits dérivés : aspects compositionnels et nutritionnels (dans le cas de l'homme). Séminaire F.I.L.-I.D.F. de Weihenstephan, 11-13 nov.

[6] Bonnet (G. F.), NepveuX (P.) (1970) - Les migraines tyraminiques. Rev. franç. Allergol., 10, 3, 233-236.

[7] BRown (H. H.) (1959). - Determination of blood urea with p-dimethylaminobenzaldehyde. Anal. Chem., 31, 11, 1844-1846.

[8] Carlini (E. A.), Green (J. P.) (1963). - The measurement of histamine in brain and its distribution. Biochem. Pharmacol., 12, 1448-1449.

[9] Causeret (J.), Mocouot (G.) (1966). - Effets des traitements thermiques du lait sur sa valeur nutritive. Cah. Nut. Diét., I, 1, 31-38.

[10] Collet (J.), Sales (M.) (1971). - Le lactosérum - Origine et composition Technologie - Utilisation dans l'alimentation du veau et du porc. Mémoire de fin d'études E.N.S.A., Rennes.

[11] Craig (J. C.), Aceto (N. C.), Della Monica (E. S.) (1961). - Occurence of 5 hydroxymethylfurfural in vacuum foamdried whole milk and its relation to processing and storage. J. Dairy Sci., 44, 10, 1827-1835.

[12] Della Monica (E.S.), Craig (J. C.), Calhoun (M. J.) (1968). - Error in the analysis of hydroxymethylfurfural in processed milk. J. Dairy Sci., 51, 3, 352-355.

[13] Efron (M. L.) (1965). - Quantitative estimation of aminoacids in physiological fluids using a Technicon analyser. Automation in analytical Chemistry Symposia,

[14] FERRARI (A.) (1960). - Nitrogen determination by a continuous digestion and analysis system. Annals of the New York academy of sciences, 87, 2, 792-800.

[15] Février (C.) (1972). - Réunion A.F.T.A.A., Grignon.

[16] GaRnier (J.) (1964). - Mise au point sur la séparation, l'identification et le dosage des protéines du lait de vache à l'exclusion cies caséines. Ann. Biol. anim. Bioch. Biophys., 4, 2, 163-187.

[17] Ghadimi (H.), Pecora (P.) (1963). - Free amino acids of differents kinds of milk. Am. J. Clin. Nutr., 13, 75-81.

[18] Gravert (H. O.) (1974). - La production du lait entre la saturation du marché et la situation excédentaire. Kieler Milchwirtschaftliche Forschungsberichte, 26, 2, 133-143.

[19] IEnistea (C.) (1971). - Bacterial production and destruction of histamine in foods and food poisoning caused by histamine. Nahrung, 15, 1, 109-113.

[20] Javillier (M.), Polonovski (M.), Florkin (M.), Boulanger (P.), Lemoigne (M.), Roche (J.), Wurmser (R.) (1959). - Traité de Biochimie générale. Tome I (premier et deuxième fascicules). 
[21] Javillier (M.), Polonovski (M.), Florkin (M.), Boulanger (P.), Lemoigne (M.), Roche (J.), Wurmser (R.) (1967). - Traité de Biochimie générale. Tome III (troisième fascicule).

[22] Kayalep (S. O.), Renda (N.), Kaymakcalan (S.), Ozer (A.) (1970), - Tyramine content of some cheeses, Toxic. appl. Pharmac., 16, 2, 459-460.

[23] KeENEY (M.), BAsSETte (R.) (1959). - Detection of intermediate compounds in the early stages of browning reaction in milk products. J. Dairy Sci, $42,6,945-960$.

[24] Klostermeyer (H.) (1974). - La situation mondiale de protéine (un appel à la recherche laitière). Kieler Milchwirtschaftliche Forschungsberichte, 26, $2,145-158$.

[25] Von KnaUt (T.) (1967). - Die proteolytischen Eigenschaften der Pseudomonas-Arten beim Wachstum in der Milch. Milchwissenschaft, 22, 5, 289-292.

[26] Kremzner (Z. L. T.), Pfeiffer (C. C.) (1966). - Identification of substances interfering with the fluorometric determination of brain histamine. Biochem. Pharmacol., 15, 197-200.

[27] De Koning (P. J.) (1968). - A new method for the fluorometric determination of histamine in cheese. Neth. Milk \& Dairy J., 22, 153-157.

[28] Lenoir (J.), Choisy (C.), Do NGoc (M.) (1971). - Les acides aminés libres des fromages affinés de Camembert, Saint-Paulin et Gruyère de Comté. Rev. Lait. Fr., 288, 447-462.

[29] LySTER (R. L. J.) (1972). - Reviews of the progress of dairy science. Section C. Chemistry of milk proteins. J. Dairy Res., 39, 279-318.

[30] Mc Dowel. (A. K. R.) (1972). - Seasonal variations in the total nitrogen, non-protein nitrogen and urea nitrogen contents of Friesian and Jersey milk. I. Dairy Res., 39, 27-33.

[31] Mc Kenzie (H. A.) (1970). - Milk proteins chemistry and molecular biology. Academic Press, New York and London.

[32] Mardens (Y.), van SAnde (M.), CAers (J.) (1971). - Ion exchange chromatographic behavior of ninhydrin positive substances. Analytical Letters, 4, 5, 285-294.

[33] Nicaise (A. M.) (1969). - L'émission en fluorescence, fondement moderne de l'exploration du métabolisme des amino-acides. Prod. et Prob. Pharm., $24,561-570$ et $24,11,621-629$.

[34] Oates (J. A.) (1961). - Methods of Medical Research. Year Book Med. Publ., Chicago (Illinois), vol. IX, 169.

[35] Petit (L.), Adrian (J.) (1967). - La réaction de Maillard. Description et répercussions physiologiques. Cah. Nut. Diét., II, 4.

[36] Phillips (R. E.), Elevitch (F. R.) (1970). - Fluorometric technics in clinical pathology and their interpretation. Progr. Clin. Path., 1, 63-153.

[37] Plumas (B.), Sautier (C.) (1972). - Méthode de dosage simultané de l'histidine et de l'histamine dans les produits biologiques. Application aux vins. Ann. Fals. Exp. Chim., 703, 322-336.

[38] PокRovsky (A. A.) (1969) - Théorie d'une alimentation équilibrée et rôle des produits laitiers dans la satisfaction des besoins nutritionnels de 1'homme. Rev. Lait. Fr, 271, 24, 803-809.

[39] Pondaven (A.) (1974). - Etude de l'évolution de la composition des lactosérums au cours de la conservation. Thèse d'Ingénieur C.N.A.M., Paris.

[40] Price (K.), SMith (S. E.) (1971). - Cheese reaction and tyramine. The Lancet, 1, 130-131.

[41] Razemon-Pinta (M.) (1971). - Les acides aminés libres du lait (acides et neutres). Thèse pour le doctorat en médecine, Lille. 
[42] RHo (J. H.) (1972). - Direct fluorometric determination of urea in urine. Clin. Chem. U.S.A., 18, 5, 476-478.

[43] Robin (P.), Robin (D.), JacQuot (R.) (1967). - Submicrodétermination des acides aminés. Bull. Soc. Chim. Biol., 49, 4, 449-451.

[44] Ruff (F.), Saindelle (A.), Dutripon (E.), Parrot (J. L.) (1967). - Automatisation du dosage fluorométrique de l'histamine. Technicon Symposium Européen, "Automatisation en chimie analytique ", Brighton, 13-15 nov.

[45] Samuelsson (E. G.), Per Nielsen (1970). - Formation of hydroxymethylfurfural caused by heat treatment of milk. Milchwissenschaft, 25, 9, 541-543.

[46] Serres (L.), Amariglio (S.), Petransxiene (D.) (1973). - Contrôle de la qualité des produits laitiers. Tome : Analyses physiques et chimiques, $2^{e}$ édition.

[47] Shore (A.), Burkhalter (A.), CoHn (V. H.) (1959). - A method for the fluorometric assay of histamine in tissues. J. Pharm. and Exper. Therap, 127, $182-186$.

[48] Spackman (D. H.), Stein (W. H.), Moore (S.) (1958), - Automatic recording apparatus for use in the chromatography of amino acids. Anal. Chem., 30, 1190.

[49] Spector (S.), Melmon (K.), Lovenberg (W.), Sjoerdsma (A.) (1963). - The presence and distribution of tyramine in mammalian tissues. J. Pharm. and Exper. Therap., 140, 229-235.

[50] Tarodo DE LA Fuente (B.) (1971). - Physicochimie de la séparation du caillé et du sérum. Tech. Lait., 719, 79-83.

[51] UdENFRIEND (S.) (1962). - Fluorescence assay in biology and medecine. Tome 1, Academic Press, New York and London.

[52] De Vuyst (A.), Vervack (W.), Charlier (H.), Jadin (V.) (1971). - L'influence du traitement thermique sur la valeur biologique de la protéine des aliments. Bull. Acad. R. Belg., 11, 2, 231-246.

[53] WatT (G. W.), ChrisP (J.D.) (1954). - Spectrophotometric method for determination of urea. Anal. Chem., 26, 452-453.

[54] Wheelock (J. V.), KNight (D. J.) (1969). - The action of rennet on whole milk. J. Dairy Res., 36, 2, 183-190.

[55] Wyeth (T. C.) (1972). - Chemical analysis of whey powders. J. Soc. Dairy Technol., 25, 3, 135-139. 